\title{
Teor e composição química do óleo essencial de erva-baleeira (Varronia curassavica Jaqc.) em função dos horários de coleta
}

QUEIROZ, T.B.'; MENDES, A.D.R.'; SILVA, J.C.R.L.'; FONSECA, F.S.A.'; MARTINS, E.R.'1

Instituto de Ciências Agrárias da Universidade Federal de Minas Gerais (ICA/UFMG), Laboratório de Plantas Medicinais, Avenida Universitária, 1.000, Bairro Universitário, Montes Claros, MG, Brasil. CEP: 39.404-547. *Autor para correspondência: tulio-bq@hotmail.com

RESUMO: A erva-baleeira (Varronia curassavica Jaqc.) é uma importante planta medicinal, com ocorrências em vários biomas brasileiros, dela é possível extrair óleo essencial utilizado na produção de fitoterápicos. O objetivo deste trabalho foi estudar o efeito do horário de coleta das folhas sobre o teor e os constituintes químicos do óleo essencial da $V$. curassavica. As folhas foram obtidas da coleção do germoplasma do Horto Medicinal do Instituto de Ciências Agrárias da Universidade Federal de Minas Gerais (ICA/UFMG). Os tratamentos constaram de cinco horários de coleta (6:00, 9:00,12:00, 15:00 e 18:00 horas) e três repetições, utilizando o delineamento experimental inteiramente casualizado. O óleo essencial foi extraído pelo método de hidrodestilação, utilizando-se o sistema Clevenger e a constituição química determinada por cromatografia em fase gasosa acoplada à espectrometria de massas (CG/EM). O teor do óleo essencial não sofreu influencia do horário de coleta. Entretanto, o horário de coleta influenciou a composição química do óleo essencial de erva-baleeira. Os compostos majoritários em todos os horários de coleta foram $\beta$-cariofileno $(22,6$ a $27,4 \%)$, elixeno $(14,9$ a $17,2 \%)$ e $\gamma$-muuroleno $(9,1$ a $9,8 \%)$. Já o composto de menor abundância relativa, sabineno $(1,1 \%)$, apresentou ocorrência apenas as $6 \mathrm{~h}$ da manhã.

Palavras-chave: Planta medicinal, caracterização química, monoterpenos, $\beta$-cariofileno, a-humuleno.

\begin{abstract}
Content and chemical composition of the essential oil of 'erva-baleeira' (Varronia curassavica Jaqc.) as a function of harvesting times. The erva-baleeira (Varronia curassavica Jacq.) is an important medicinal plant which occurs in several Brazilian biomes, from which it is possible to extract essential oil used in the production of herbal medicines. The objective of this study was to observe the effect of the collection schedule for the leaves on the content and the chemical constituents of the essential oil of $V$. curassavica. The leaves were obtained from the germplasm collection of the Medicinal Garden of the Institute of Agricultural Sciences at the Federal University of Minas Gerais (ICA/UFMG).

The treatments consisted of five collection schedules (6:00 and 9:00 AM, and 12:00, 3:00, and 6:00 PM) and three replications, using a completely randomized design. The essential oil was extracted by the hydrodistillation method, using the Clevenger system, and the chemical composition was determined by gas chromatography with mass spectrometry (GC/MS). The harvest time did not influence the content of the essential oil. However, the collection schedule did influence the chemical composition of the essential oil of erva-baleeira. The major compounds at all collection times were $\beta$-caryophyllene (22.6 to $27.4 \%$ ), xylene (14.9 to $17.2 \%$ ) and $y$-muurolene (9.1 to $9.8 \%)$. The compound of lowest relative abundance, sabinene $(1.1 \%)$, was found only at 6 AM.
\end{abstract}

Keywords: medicinal plant, chemical characterization, monoterpenes, $\beta$-caryophyllene, a-humulene. 


\section{INTRODUÇÃO}

Varronia curassavica Jacq., pertencente a família Boraginaceae é conhecida popularmente como erva baleeira, cuja sinonímia é Cordia verbenacea DC, é uma planta medicinal arbustiva, amplamente distribuída no Brasil (Miller \& Gottschling, 2007; Lorenzi \& Matos, 2008). Está presente na mata atlântica, regiões baixas da Amazônia (Lorenzi, et al., 2003), cerrado e caatinga (Mendes et al, 2015). Suas propriedades medicinais estão atribuídas ao óleo essencial presente nas folhas, que apresenta ação anti-inflamatória, cicatrizante e antiulcerogênica devido à presença de mono e sesquiterpenos, dentre os quais destacamse o $\alpha$-humuleno e $\beta$-cariofileno (Carvalho Junior et al., 2004). Tais características possibilitaram desenvolver, a partir do óleo essencial obtido dessa espécie, o primeiro fitoterápico brasileiro de uso tópico, com ação anti-inflamatória (Quispe-Condori et al., 2008; Ryan, 2010).

A V. curassavica consta em diferentes listas oficiais do Ministério da Saúde no Brasil, sendo utilizada na preparação de medicamentos fitoterápicos e/ou drogas vegetais industrializadas (Carvalho et al., 2008; Renisus, 2009). As drogas vegetais consistem em plantas medicinais ou suas partes que apresentem substâncias terapêuticas após o processo de coleta (Brasil, 2010). No entanto, o teor e a composição química dessas substâncias podem apresentar variações ao longo do dia, sendo o horário de coleta um relevante parâmetro para a produção de óleo essencial (Souza et al., 2006). Assim, fatores abióticos também são responsáveis por influenciar a produção de metabólitos secundários, com consequente efeito na qualidade da matéria prima (Gobbo Neto \& Lopes, 2007). Dentre estes fatores tem-se, a fertilização do solo, a intensidade luminosa (Pegoraro et al., 2010), a lâmina de irrigação (Lopes et al., 2011), o intervalo e a época de colheita (Figueiredo et al., 2009; Blank et al., 2012).

O efeito do horário de coleta sobre o teor e as proporções relativas dos compostos químicos do óleo essencial tem sido estudado por diversos pesquisadores (Blank et al., 2005, 2007; Nascimento et al., 2006; Melo et al., 2011; Ehlert et al., 2013). Esses estudos mostram que não existe um padrão para as variações durante o ciclo dia/noite nas concentrações de óleos voláteis, portanto, cada espécie apresenta uma variação específica.

Com o intuito de estabelecer boas práticas de coleta das folhas de erva-baleeira o objetivo desse trabalho foi avaliar o efeito do horário de coleta das folhas sobre o teor e os constituintes químicos do óleo essencial da $V$. curassavica.

\section{MATERIAL E MÉTODO}

O experimento foi conduzido no Laboratório de Plantas Medicinais e Aromáticas do Instituto de Ciências Agrárias da Universidade Federal de Minas Gerais (ICA/UFMG), Montes Claros, MG. As plantas utilizadas neste estudo pertencem à coleção de germoplasma de erva-baleeira do Horto Medicinal do ICA/UFMG. A escolha do acesso ICA-VC4, ocorreu devido à presença majoritária do composto $\beta$-cariofileno, característica agronômica e química desejada em plantios comerciais para atender a demanda da indústria farmacêutica.

A coleção de germoplasma de erva-baleeira está localiza a $16^{\circ} 40^{\prime} 58,5^{\prime \prime}$ de latitude sul, $43^{\circ} 50^{\prime}$ $25,6^{\prime \prime}$ de longitude oeste de Greenwich e altitude 626 metros, ao norte do estado de Minas Gerais. O clima local, segundo a classificação de Köppen, é do tipo Aw tropical quente úmido, com duas estações bem definidas, verão quente e chuvoso e o inverno frio e seco. Os dados climatológicos foram obtidos nos horários do dia de coleta, pela estação meteorológica automática do GEMISA - Grupo de estudos em manejo e irrigação no Semiárido - do ICA/UFMG, localizada a aproximadamente a $20 \mathrm{~m}$ da coleção de germoplasma.

As plantas de erva-baleeira foram mantidas sob irrigação por gotejamento, em solo Argiloso Vermelho-Amarelo, com as características químicas e físicas na camada de $0-20 \mathrm{~cm}$ de profundidade conforme Tabela 1.

O espaçamento de cultivo foi de $1,00 \mathrm{~m} \mathrm{x}$ $0,50 \mathrm{~m}$. As exsicatas da coleção de germoplasma foram encaminhadas ao Instituto de Biociências, Letras e Ciências Exatas da Universidade Estadual Paulista (IBILCE/UNESP) para identificação taxonômica pela Profa. Dra. Neusa Taroda Ranga. As exsicatas encontram-se armazenadas no herbário-didático do ICA/UFMG.

O delineamento experimental foi inteiramente casualizado, com cinco tratamentos,

TABELA 1. Características químicas e físicas do solo típico de cerrado da Região de Montes Claros - MG, do banco de germoplasma de Varronia Curassavica Jacq

\begin{tabular}{|c|c|c|c|c|c|c|c|c|c|c|c|c|c|}
\hline \multicolumn{13}{|c|}{$\mathrm{H}_{2} \mathrm{O}$} & Argila \\
\hline & $\mathrm{mmol}_{\mathrm{c}} \mathrm{dm}^{-3}$ & -----. & ---- & $-\mathrm{cmol}$ & $n^{-33-}$ & & & $\%$ & -----' & -----Da & kg-1-1--1 & & \\
\hline 6,9 & 10 & 6,9 & 1,9 & 0 & 8,8 & 10,46 & 0 & 84 & 3,39 & 9 & 25 & 34 & 32 \\
\hline
\end{tabular}

Rev. Bras. PI. Med., Campinas, v.18, n.1, supl. I, p.356-362, 2016. 
que foram os horários de coleta $(6: 00,9: 00,12: 00$, 15:00 e 18:00 horas) e três repetições. Foram analisadas as seguintes características: teor de óleo essencial e identificação dos constituintes químicos do óleo essencial de erva-baleeira.

Em cada horário, amostras das folhas sadias (40 g) da porção mediana do acesso ICAVC4, foram coletadas em triplicata, acondicionadas em sacos plásticos e conduzidas ao laboratório de Plantas medicinais e aromáticas do ICA/UFMG. A extração do óleo essencial, do material vegetal fresco, foi realizada por hidrodestilação no aparelho de Clevenger, durante duas horas (AOAC, 1995). O teor de óleo essencial foi expresso com base na matéria seca da amostra, que foram mantidas após extração do óleo essencial em estufa com circulação forçada de ar a $65^{\circ} \mathrm{C}$, até peso constante.

A análise da composição química do óleo essencial foi realizada em triplicata Laboratório de Química Instrumental do ICA/UFMG. As amostras de óleos essenciais, de todos os tratamentos, foram analisadas individualmente por cromatografia em fase gasosa acoplada a espectrometria de massas (CG-EM). Utilizou-se sistema Agilent Technologies (7890A) acoplado ao espectrômetro de massas (MS 5975C). Foi utilizada coluna capilar HP-5ms (30 m x $0,25 \mathrm{~mm} \times 0,25 \mu \mathrm{m}$ ) e hélio (fluxo $1 \mathrm{~mL} \mathrm{~min}^{-1}$ ) como gás de arraste. $O$ injetor mantido a $220^{\circ} \mathrm{C}$, seguindo a programação da temperatura de $60^{\circ} \mathrm{C}-240^{\circ} \mathrm{C}$ $\left(3^{\circ} \mathrm{C} \mathrm{min}^{-1}\right)$ operando em impacto eletrônico a $70 \mathrm{eV}$, em uma faixa de 40 a $550(\mathrm{~m} / \mathrm{z})$. Os cálculos dos índices de retenção foram baseados na injeção do padrão de $n$-alcanos ( $n \mathrm{C} 8-n \mathrm{C} 40)$.
A identificação dos constituintes do óleo essencial foi realizada através do software MSD Chemstation, juntamente com a biblioteca (NIST - National Institute of Standards and Technology, USA). A abundância relativa (\%) dos constituintes foi calculada a partir da área de pico do cromatograma de íons totais (TIC) e organizada de acordo com a ordem de eluição. A identificação do $\beta$-cariofileno e do $\alpha$-humuleno foi realizada pela injeção do padrão sintético (Sigma Aldrich) e os demais compostos por comparação do espectro de massas da biblioteca (NIST 2.0). O índice de retenção (IR) relativo de cada composto foi calculado segundo Van den dool \& Kratz (1963) e comparado com informações da literatura (Adams, 2012).

O teor de óleo essencial foi submetido à análise estatística pelo programa SAEG - Sistema para Análises Estatísticas e Genéticas (Ribeiro Júnior, 2001), através da análise de variância e o teste de média Scott Knott, a 5\% de probabilidade. Os elementos climáticos radiação solar, temperatura, a umidade relativa do ar e a velocidade do vento foram avaliados por regressão múltipla.

\section{RESULTADO E DISCUSSÃO}

Os teores médios de óleo essencial de ervabaleeira, obtido nos diferentes horários de coleta, não apresentaram diferença significativa (Figura 1). No entanto, Souza et al. (2011), verificaram maior teor de óleo essencial nas folhas de erva-baleeira coletadas às 18:00. Tal comportamento pode ser explicado, devido à variação entre os genótipos

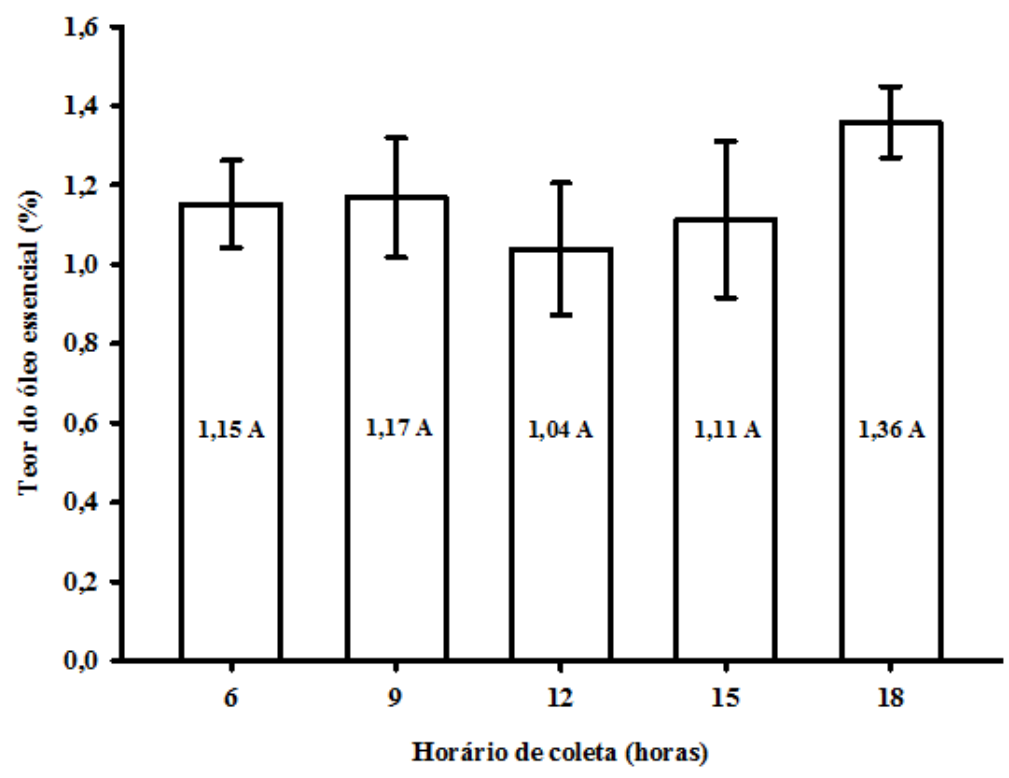

FIGURA 1. Teor do óleo essencial extraído de folhas de Varronia curassavica, coletadas em diferentes horários (as barras indicam o desvio padrão e as médias seguidas das mesmas letras indicam semelhança pelo teste Scott Knott a $5 \%$ de probabilidade) 
analisados em cada um dos estudos. Rosa et al., (2010) estudando dois genótipos de palmarosa (Cymbopogon martinii) observaram, que existe variação no rendimento do óleo essencial e que a coleta das folhas dessa espécie pode ser feita tanto pela manhã como pela tarde. Além disso, o teor de óleo essencial pode ser influenciado pela variação sazonal, que estimula produção diferenciada ao logo do ano. Botreal et al. (2010), estudando o teor e a composição química do óleo essencial da Hyptis marrubioides Epl., em função da sazonalidade, não observou diferença qualitativa do óleo essencial nas diferentes estações, contudo verificou que existe diferença na abundância dos compostos químicos presentes no óleo essencial.

No trabalho de Ehlert et al. (2013) foi constatado que o teor de óleo essencial de ervacidreira brasileira (Lippia alba (Mill.) N. E. Br.), em função do horário de coleta, não apresentou diferença significativa para os horários (8:00, 10:00, 12:00, 14:00 e 16:00 horas). Dados semelhantes também foram observados no trabalho de Blank et al. (2005) que, estudando a melissa (Melissa officinalis L.), em diferentes horários de coleta (9:00 e 15:00 horas), não observaram influência no teor de óleo essencial. No entanto, Melo et al., (2011) avaliando o óleo essencial de alecrim-pimenta (Lippia sidoides Cham.) observaram que o horário de coleta afetou o teor do óleo essencial, sendo recomendado o horário em torno de 10:00 horas, visando à obtenção do maior teor de óleo essencial. As espécies medicinais produtoras de

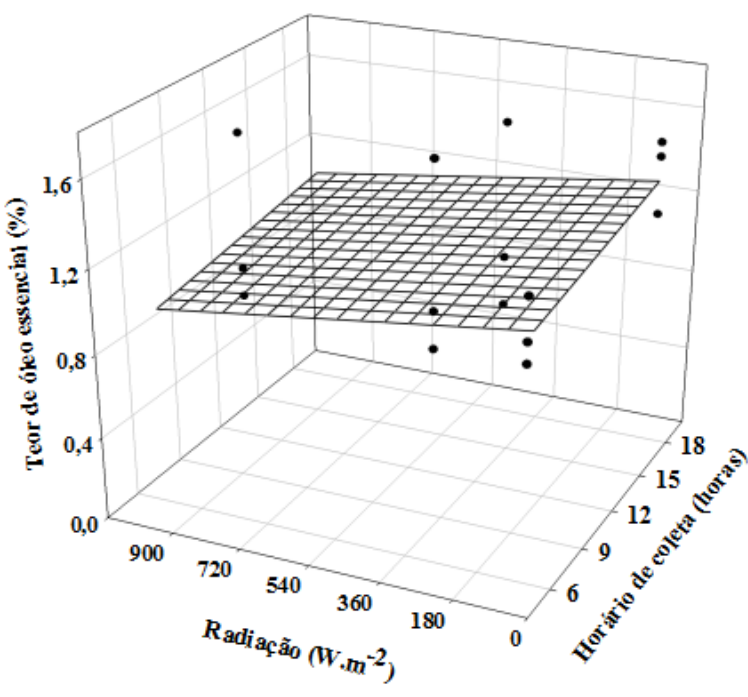

Teor $=-0,0002^{\text {ns }} H_{0 r a}{ }^{2}+0,0118^{\text {ns }}$ Radiação+1,1135 ${ }^{\text {ns }} R^{2}=0,30^{\text {ns }}$

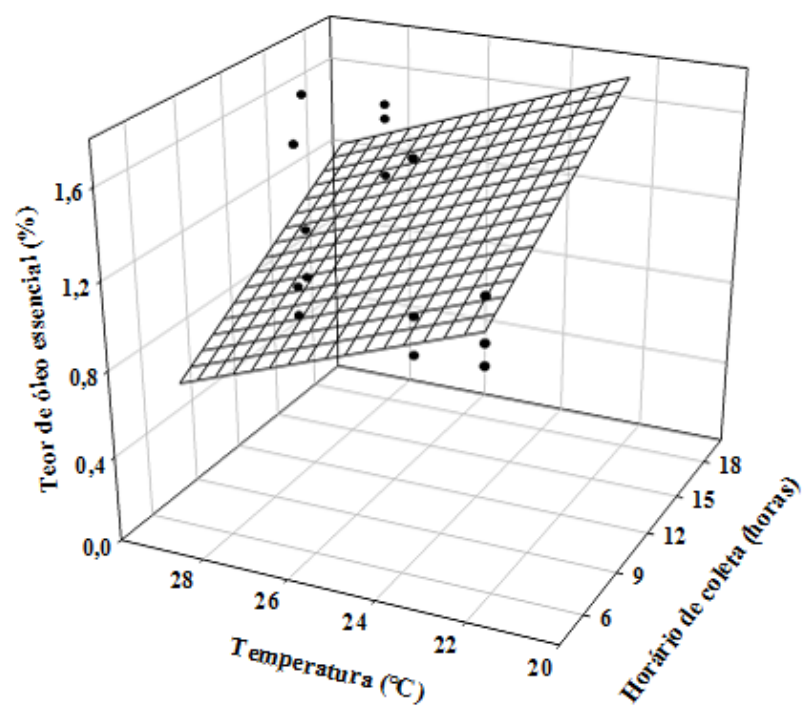

Teor $=-0,00713^{\text {ns }}$ Hora $^{2}+0,0503^{n s}$ Temp eratura $+2,4487^{n s} R^{2}=0,34^{\text {ns }}$

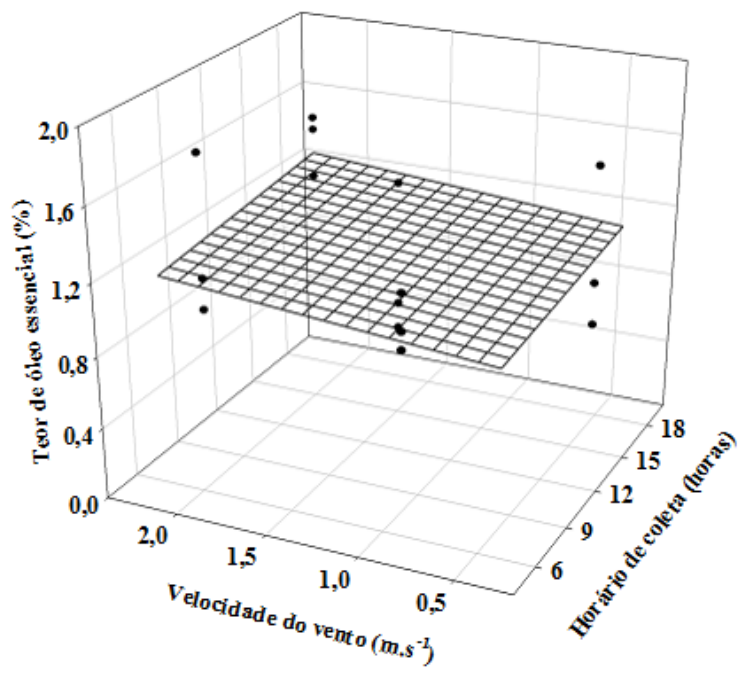

Teor $=0,0806^{\mathrm{ns}}$ Hora $^{2}+0,0099^{\mathrm{ns}}$ Veloc. do vento $+0,9348^{\mathrm{ns}} \mathrm{R}^{2}=0,06^{\mathrm{ns}}$

FIGURA 2. Análise de regressão múltipla entre os valores médios do teor de óleo essencial (\%), as condições climáticas [A: Radiação $\left(\mathrm{W} \mathrm{m}^{-2}\right)$ B: temperatura $\left(\mathrm{W} \mathrm{m}^{-2}\right)$ e C: velocidade vento $\left(\mathrm{W} \mathrm{m}^{-2}\right)$ ] e o horário de coleta $(\mathrm{h})$

Rev. Bras. PI. Med., Campinas, v.18, n.1, supl. I, p.356-362, 2016. 
TABELA 2. Abundância relativa média dos constituintes químicos (\%) seguido do \pm desvio padrão do óleo essencial de erva-baleeira (Varronia curassavica), analisada por CG-EM em diferentes horários de coleta

\begin{tabular}{|c|c|c|c|c|c|c|c|}
\hline \multirow{2}{*}{ Compostos } & \multirow{2}{*}{$\mathrm{IR}^{\mathrm{c}}$} & \multirow{2}{*}{ IRr } & \multicolumn{4}{|c|}{ Área cromatograma (TIC\%)* } & \multirow[b]{2}{*}{$18: 00$} \\
\hline & & & $6: 00$ & 9:00 & $12: 00$ & $15: 00$ & \\
\hline a-Pineno & 933 & 939 & $8,4 \pm 1,3$ & $6,0 \pm 2,7$ & $7,1 \pm 1,7$ & $8,1 \pm 0,8$ & $9,5 \pm 1,8$ \\
\hline Sabineno & 972 & 975 & $1,1 \pm 0,3$ & - & - & - & - \\
\hline$\beta$-Pineno & 979 & 979 & $4,0 \pm 0,6$ & $3,1 \pm 1,1$ & $3,8 \pm 0,4$ & $3,9 \pm 0,5$ & $4,3 \pm 0,8$ \\
\hline ঠ-3-Careno & 1032 & 1033 & $1,4 \pm 0,2$ & $1,2 \pm 0,3$ & $1,2 \pm 0,2$ & $1,3 \pm 0,3$ & $1,4 \pm 0,3$ \\
\hline$\beta$-Bourboneno & 1380 & 1380 & $1,6 \pm 0,0$ & $1,8 \pm 0,1$ & $1,7 \pm 0,2$ & $2,0 \pm 0,4$ & $1,7 \pm 0,0$ \\
\hline$\beta$-Elemeno & 1386 & 1391 & $2,8 \pm 0,2$ & $3,2 \pm 0,2$ & $3,5 \pm 0,3$ & $3,5 \pm 0,7$ & $3,2 \pm 0,1$ \\
\hline$\beta$-Cariofileno & 1417 & 1419 & $23,3 \pm 1,3$ & $27,4 \pm 2,9$ & $24,4 \pm 0,2$ & $25,1 \pm 6,1$ & $22,6 \pm 0,5$ \\
\hline Aristoleno & 1438 & 1446 & $4,6 \pm 0,3$ & $3,9 \pm 0,3$ & $4,6 \pm 0,2$ & - & $4,6 \pm 0,2$ \\
\hline a-Humuleno & 1451 & 1455 & $4,4 \pm 0,2$ & $4,8 \pm 0,3$ & $3,3 \pm 0,7$ & $4,7 \pm 0,9$ & $4,3 \pm 0,1$ \\
\hline Y-Muuroleno & 1478 & 1477 & $9,1 \pm 0,2$ & $9,8 \pm 0,6$ & $9,4 \pm 0,6$ & $9,7 \pm 1,9$ & $9,7 \pm 0,2$ \\
\hline Elixeno & 1491 & 1498 & $15,5 \pm 0,4$ & $17,2 \pm 2,1$ & $14,9 \pm 1,6$ & $15,2 \pm 1,3$ & $16,3 \pm 0,5$ \\
\hline Espatulenol & 1572 & 1578 & $3,2 \pm 0,3$ & $3,1 \pm 0,3$ & $3,8 \pm 0,4$ & $4,3 \pm 0,8$ & $2,1 \pm 1,8$ \\
\hline Óxido de cariofileno & 1577 & 1583 & $1,8 \pm 0,1$ & $1,8 \pm 0,2$ & $2,1 \pm 0,4$ & $2,3 \pm 0,7$ & $1,6 \pm 0,2$ \\
\hline Compostos traços & & & 18,9 & 16,4 & 20 & 19,7 & 18,8 \\
\hline Monoterpenos & & & 14,8 & 10,3 & 12,1 & 13,3 & 15,2 \\
\hline Sesquiterpenos & & & 61,3 & 68,4 & 62 & 60,5 & 62,3 \\
\hline Sesquiterpenos oxigenados & & & 5 & 4,9 & 5,9 & 6,5 & 3,7 \\
\hline Teor de óleo (\%) & & & 7,7 & 5,2 & 4,7 & 6,3 & 8,2 \\
\hline
\end{tabular}

Área cromatograma $(\mathrm{TIC} \%)^{*}$ :Essa área é correspondente aos íons totais dos compostos detectados, deste modo para alguns compostos essa área não pode ser considerada um parâmetro de quantificação, (-): composto não detectado, TR: Tempo de retenção, IR: índice de retenção calculado, IRr: índice de retenção da literatura, Foram considerados compostos traços aqueles com abundância relativa menor a $1 \%$ da área do pico do cromatograma.

óleo essencial apresentam diferentes respostas ao horário de coleta. Por esta razão, se faz necessário ampliar cada vez mais os estudos com essas plantas para determinar qual o melhor horário de coleta de cada espécie em cada estação do ano (Ehlert et al., 2013). Apesar de Souza et al. (2011), proporem um horário de coleta para a $V$. curassavica, estes autores não apresentaram a composição química do óleo essencial ao longo dia.

O horário da coleta não atua isoladamente sobre o metabolismo da planta, fatores como temperatura, disponibilidade hídrica, radiação solar, nutrientes minerais, altitude, vento, umidade relativa do ar e poluição atmosférica também afetam o metabolismo primário e secundário (Gobbo Neto \& Lopes, 2007; Souza et al., 2011).

Nos horários de coleta, pela análise de regressão múltipla (Figura 2), verificou-se que os elementos climáticos: radiação solar, temperatura, a umidade relativa do ar e a velocidade do vento, não influenciaram significativamente o teor de óleos voláteis das folhas de erva-baleeira.

A análise cromatográfica (CG-EM) dos óleos essenciais extraídos das folhas de ervabaleeira permitiu a identificação de treze compostos, perfazendo a maior parte $(80 \%$ a $83,6 \%)$ da composição do óleo essencial (Tabela 2). Esses óleos são ricos em sesquiterpenos $(60,5 \%$ a $68,4 \%$ ) e monoterpenos (10,3\% a $15,2 \%)$, sendo os sesquiterpenos funcionalizados correspondem à menor parte dos compostos identificados $(3,7 \%$ a $6,51 \%)$. O $\beta$-cariofileno $(22,6 \%$ a $27,4 \%)$ foi o composto majoritário em todas as amostras analisadas, seguido do elixeno $(14,9 \%$ a $17,2 \%)$ e do $y$-muuroleno $(9,1 \%$ a $9,8 \%)$. Dos monoterpenos, o sabineno $(1,1 \%)$ foi detectado apenas no primeiro horário de coleta (6:00 horas), enquanto o aristoleno não foi detectado às 15:00 horas. Ambos os compostos químicos estão associados à atividade bactericida (Shimizu et al., 2006; Karamian et al., 
2013).

O $\beta$-cariofileno e o $\alpha$-humuleno são os dois principais sesquiterpenos responsáveis pela atividade anti-inflamatória do óleo essencial da erva-baleeira (Fernandes et al., 2007). No presente estudo, o horário de coleta não influenciou na abundância relativa do $\beta$-cariofileno (22,6\% a 27,4\%) e do $\alpha$-humuleno (3,3 \% a 4,8 \%). Esses valores estão de acordo com os encontrados por Carvalho Junior et al. (2004) e Rodrigues et al. (2012), que observaram valores semelhante para o $\beta$-cariofileno $(25,27 \%$ e $25,4 \%)$ para o $\alpha$-humuleno $(4,8 \%$ e 4,64 $\%)$. O teor $\alpha$-humuleno nos horários de coleta atingiu valores superiores ao teor mínimo $(2,3 \%)$ necessário para o uso como matéria-prima em medicamentos fitoterápicos (Quispe-Condori et al., 2008).

Conclui-se que o horário de coleta associado às diferentes condições climáticas não apresentou efeito significativo no teor de óleo essencial da $V$. curassavica e na abundância relativa dos compostos químicos $\beta$-cariofileno e $\alpha$-humuleno, presentes no óleo essencial. Além disso, a composição química do óleo essencial apresentou variação nos diferentes horários de coleta, com restrição da presença dos compostos sabineno e aristoleno em horários específicos.

\section{AGRADECIMENTOS}

Os autores agradecem ao Laboratório de Química Instrumental e de Plantas Medicinais e Aromáticas do ICA-UFMG e ao Programa de Educação Tutorial, PET-Agronomia ICA-UFMG.

\section{REFERÊNCIAS}

ADAMS, R. P. Identification of essential oils componets by gás chromatography/ mass spectroscopy. 4.ed. Allured Bussiness Media, USA, 2012. 804p.

ASSOCIATION OF OFFICIAL ANALYTICAL CHEMISTS. Official methods of analysis. 16.ed. Arlington: AOAC, 1995. $1141 \mathrm{p}$

BLANK, A. F. et al. Influence of season, harvest time and drying on Java citronella (Cymbopogon winterianus Jowitt) volatile oil. Brazilian Journal of Pharmacognosy, v.17, p. 557-564, 2007.

BLANK, A. F. et al. Influência do horário de colheita e secagem de folhas no óleo essencial de melissa (Melissa officinalis L.) cultivada em dois ambientes. Revista Brasileira de Plantas Medicinais, v.8, n.1, p.73-78, 2005.

BLANK, A. F. et al. Espaçamento de plantio e intervalos de colheita na biomassa e no óleo essencial de gerânio. Horticultura Brasileira, v. 30, n. 4, p. 740-746, 2012.

BOTREAL, P. P. et al. Teor e composição química do oleo essencial de Hyptis marrubioides Epl., Lamiaceae em função da sazonalidade. Acta Scientiarum. Agronomy, v. 32, n. 3, p. 533-538, 2010.
BRASIL. Ministério da Saúde. Agência Nacional de Vigilância Sanitária. RDC $\mathbf{n}^{\circ} .10$ de 10 de março de 2010. Dispõe sobre a notificação de drogas vegetais junto à Agência Nacional de Vigilância Sanitária (ANVISA) e dá outras providências. ANVISA; 2010. Relação Nacional de plantas medicinais de interesse ao SUS (RENISUS). 2009. http://bvsms.saude.gov.br/ bvs/sus/pdf/marco/ms_relacao_plantas_medicinais_ sus 0603.pdf. Acesso em 12 de nov. 2015.

CARVALHO JUNIOR, P.M. et al. Chemical composition and antimicrobial activity of the essential oil of Cordia verbenacea DC. Journal of Ethnopharmacology, v. 95, p. 297-301, 2004.

CARVALHO, A. C. B. et al. Situação do registro de medicamentos fitoterápicos no Brasil. Revista Brasileira Farmacognosia, v.18, p.314-319, 2008.

VAN DEN DOOL, H.; KRATZ, P. Dec. A generalization of the retention index system including linear temperature programmed gas $\square$ liquid partition chromatography. Journal of Chromatography A, v. 11, p. 463-471, 1963.

EHLERT P. A. D. et al. Influência do horário de colheita sobre o rendimento e composição do óleo essencial de erva-cidreira brasileira [Lippia alba (Mill.) N. E. Br.]. Revista Brasileira de Plantas medicinais, v. 15, p. 72-77, 2013.

FERNANDES, E. S. et al. Anti-inflamatory effects of compounds alpha-humulene and (-)-trans-caryophyllene isolated from the essential oil of Cordia verbenacea. European Journal of Pharmacology, v. 27, p. 228236, 2007.

FIGUEIREDO, L. S. et al. Efeito da época de colheita na produção de fitomassa e rendimento de óleo essencial de alecrim-pimenta (Lippia sidoides Cham.). Revista Brasileira de Plantas Medicinais, v.11, n.2, p.154158, 2009.

GOBBO NETO, L.; LOPES, N. P. Plantas Medicinais: fatores de influência no conteúdo de metabólitos secundários. Química Nova, v. 30, p. 374-81, 2007.

LOPES, O. D. et al. Determinação do coeficiente de cultura (Kc) e eficiência do uso de água do alecrim-pimenta irrigado. Revista Brasileira de Engenharia Agrícola e Ambiental, v.15, n.6, p.548-553, 2011.

LORENZI, H.; MATOS, F. J. A. Plantas medicinais no Brasil: nativas e exóticas. 2. ed. Nova Odessa: Instituto Plantarum, 2008. 544p.

LORENZI, H.; SOUZA, M.A. TORRES; V.L.B. BACHER. Árvores exóticas no Brasil: Madeireiras, ornamentais e aromáticas. 1. ed. Nova Odessa: Instituto Plantarum, 2003. 384p.

MELO, M. T. P. et al. Teor de óleo essencial de alecrimpimenta em função do horário de colheita. Ciência Rural, v. 41, p. 1166-1169, 2011.

MENDES, A. D. R. et al. Ecogeografia de populações de erva-baleeira (Varronia curassavica) no Norte e Vale do Jequitinhonha em Minas Gerais. Ciência Rural, v.45, n.3, p.418-424, 2015

MILLER, J. S.; GOTTSCHLING, M. Generic classification in the Cordiaceae (Boraginales): resurrection of the genus Varronia P. Br. Táxon, v.56, p.163-169, 2007.

KARAMIAN, R. et al. Essential oil compositions and in vitro antioxidant and antibacterial activities of the methanol extracts of two Salvia species (Lamiaceae)

Rev. Bras. PI. Med., Campinas, v.18, n.1, supl. I, p.356-362, 2016. 
from Iran. International Journal of Agriculture and Crop Sciences, v. 11, p. 1171-1182, 2013.

NASCIMENTO, I. B. et al. Influência do horário de coleta na produção de óleo essencial de capim santo (Andropogum sp.). Revista Caatinga, v.19, p. 123-127, 2006.

PEGORARO, R. L. et al. Produção de óleos essenciais em plantas de Mentha x piperita var. piperita (Lamiaceae) submetidas a diferentes níveis de luz e nutrição do substrato. Revista Brasileira de Botânica, v.33, p.631637, 2010.

QUISPE-CONDORI, M.A. et al. Obtaining $\beta$-caryophyllene from Cordia verbenacea de Candolle by supercritical fluid extraction. The Journal of Supercritical Fluids, v. 46, p. 27-32, 2008.

RIBEIRO JÚNIOR, J. I. Análises estatísticas no SAEG. $1^{\text {a }}$. ed. Viçosa: UFV. 2001. 301p.

RODRIGUES, F.F.G. et al. Chemical composition, antibacterial and antifungal activities of essential oil from Cordia verbenacea DC leaves. Pharmacognosy Reserarch, v.4, p. 161-165, 2012.
ROSA, Y. R. S. et al. Influência do horário de colheita no óleo essencial de diferentes partes da planta de dois genótipos de palmarosa (Cymbopogon martinii). Scientia plena, v.6, n.10, pag.1-6, 2010.

RYAN, M. P. Patent Incentives, Technology Markets, and Public-Private Bio-Medical Innovation Networks in Brazil. World Development, v. 38, p. 1082-1093, 2010.

SHIMIZU M. T. et al. Óleo essencial de Lithraea molleoides (Vell.): composição química e atividade antimicrobiana. Brazilian Journal Microbiolbiology, v. 37, p. 556-560, 2006.

SOUZA, M. F. et al. Influência do horário de coleta, orientação geográfica e dossel na produção de óleo essencial de Cordia verbenaceae DC. Biotemas, v.24, p. 9-14, 2011.

SOUZA, W. P. et al. Avaliação do teor e da composição química do óleo essencial de Mentha piperita (L.) Huds durante o período diurno em cultivo hidropônico. Revista Brasileira de Plantas Medicinais, v.8, p.108111, 2006. 